\title{
Feasibility, Validity, and Reliability of the Italian Pediatric Quality of Life Inventory Multidimensional Fatigue Scale for Adults in Inpatients with Severe Obesity
}

\author{
Gian Mauro Manzoni ${ }^{a}$ b $\quad$ Alessandro Rossi ${ }^{a} \quad$ Nicoletta Marazzic \\ Fiorenza Agostic Alessandra De Col ${ }^{c}$ Giada Pietrabissa ${ }^{a}$ d \\ Gianluca Castelnuovo ${ }^{a, d}$ Enrico Molinaria, d Allessandro Sartorioc, e \\ a Istituto Auxologico Italiano IRCCS, Psychology Research Laboratory, Piancavallo, Italy; \\ ${ }^{b}$ Faculty of Psychology, eCampus University, Novedrate, Italy; ${ }^{c}$ Istituto Auxologico Italiano \\ IRCCS, Experimental Laboratory for Auxo-Endocrinological Research, Milan and Piancavallo, \\ Italy; ${ }^{d}$ Department of Psychology, Catholic University, Milan, Italy; ${ }^{\mathrm{e}}$ Istituto Auxologico \\ Italiano IRCCS, Division of Metabolic Diseases and Auxology, Piancavallo, Italy
}

Keywords

Obesity $\cdot$ Fatigue $\cdot$ Multidimensional Fatigue Scale $\cdot$ Validity $\cdot$ Reliability

\begin{abstract}
Objective: This study was aimed to examine the feasibility, validity, and reliability of the Italian Pediatric Quality of Life Inventory Multidimensional Fatigue Scale (PedsQLTM MFS) for adult inpatients with severe obesity. Methods: 200 inpatients (81\% females) with severe obesity $\left(B M I \geq 35 \mathrm{~kg} / \mathrm{m}^{2}\right.$ ) completed the PedsQL MFS (General Fatigue, Sleep/Rest Fatigue and Cognitive Fatigue domains), the Fatigue Severity Scale, and the Center for Epidemiologic Studies Depression Scale immediately after admission to a 3-week residential body weight reduction program. A randomized subsample of 48 patients re-completed the PedsQL MFS after 3 days. Results: Confirmatory factor analysis showed that a modified hierarchical model with two items moved from the Sleep/Rest Fatigue domain to the General Fatigue domain and a second-order latent factor best fitted the data. Internal consistency and test-retest reliabilities were acceptable to high in all scales, and small to high statistically significant correlations were found with all convergent measures, with the exception of BMI. Significant floor effects were found in two scales (Cognitive Fatigue and Sleep/Rest Fatigue). Conclusion: The Italian modified PedsQL MFS for adults showed to be a valid and reliable tool for the assessment of fatigue in inpatients with severe obesity. Future studies should assess its discriminant validity as well as its responsiveness to weight reduction.

(C) 2018 The Author(s)

Published by S. Karger GmbH, Freiburg
\end{abstract}


Manzoni et al.: Feasibility, Validity, and Reliability of the Italian Pediatric Quality of Life Inventory Multidimensional Fatigue Scale for Adults in Inpatients with Severe Obesity

\section{Introduction}

Fatigue is a subjective experience characterized by perceived lack of energy, physical and mental tiredness, and apathy [1,2]. It is frequently reported in the general population [3] and is a distressing symptom in a large number of medical and psychological disorders, including depression, rheumatoid disorders, stroke, multiple sclerosis, mood disorders, psychiatric disturbances, and cancer [1].

Fatigue was found to be significantly associated also with obesity in both adults and children [4-6] and has been already assessed as an outcome variable in two clinical studies on the treatment of obesity and physical correlates $[7,8]$.

Apart from the cross-sectional study of Resnick [6], in which fatigue was measured by means of only one question, three self-report multi-item questionnaires were used in all other studies involving subjects with obesity: the short form of the Multidimensional Fatigue Symptom Inventory (MFSIsf) [5], the Pediatric Quality of Life Inventory Multidimensional Fatigue Scale (PedsQL ${ }^{\mathrm{TM}}$ MFS) [4], and the Fatigue Severity Scale (FSS) [7, 8].

The FSS is one of the self-report questionnaires most commonly used for the measurement of fatigue in chronic diseases and covers the physical, social and cognitive effects of fatigue $[9,10]$. Impellizzeri et al. [11] examined the validity, reliability, and responsiveness of the Italian FSS in a sample of patients with obesity referred to a residential body weight reduction (BWR) program and concluded that 'the FSS is a short, simple, valid and reliable tool for assessing and quantifying fatigue in obese patients'. However, the FSS is a unidimensional measure, and it does not give different scores for the diverse domains of the fatigue experience. Fatigue may indeed express by various combinations of physical/neuromuscular, emotional/affective, and mental/cognitive symptoms, therefore suggesting the importance of measuring all its different manifestations [5].

Both the MFSIsf [12] and the PedsQL MFS [4] were specifically developed to tap into the multidimensionality of the fatigue experience. The former covers domains of General Fatigue (e.g., 'I feel tired'), Physical Fatigue (e.g., 'Physically I feel only able to do a little'), Activity (e.g., 'I feel very active'), Motivation (e.g., 'I dread having to do things'), and Mental Fatigue (e.g., 'My thoughts easily wander'), and is available only for adults. The latter covers three domains (Cognitive Fatigue, Sleep/Rest Fatigue, and General Fatigue), has forms designed and adapted for toddlers (2-4 years of age), young children (5-7 years), children (8-12 years), adolescents (13-18 years), young adults (18-25 years), and adults (over 26 years), and each of them has been already translated in several languages, including Italian. However, no study to date has yet assessed the psychometric properties of the Italian versions.

This study was thus aimed to understand whether the Italian version of the PedsQL MFS for adults (over 26 years) is a feasible, valid, and reliable tool for measuring the diverse domains of fatigue in inpatients with severe obesity. Specifically, we sought to examine its: i) feasibility, ii) construct validity (i.e. structural and convergent validity), iii) reproducibility (reliability, agreement and minimal detectable change), and iv) internal consistency. In particular, we hypothesized that a modified hierarchical model with two items moved from the Sleep/Rest Fatigue domain to the General Fatigue domain and a second-order latent factor fits better the data than the original model. Moreover, we expected significant correlations between: i) all the PedsQL MFS scales and the FSS but higher for the scale reflecting the second-order factor, i.e. Total Fatigue; ii) all the PedsQL MFS scales and depression; iii) General Fatigue and the number of obesity-related comorbidities; and iv) General Fatigue as well as Sleep/Rest Fatigue and BMI. 
Table 1. Socio-demographic characteristics of inpatients participating to the study
Manzoni et al.: Feasibility, Validity, and Reliability of the Italian Pediatric Quality of Life Inventory Multidimensional Fatigue Scale for Adults in Inpatients with Severe Obesity

\begin{tabular}{ll}
\hline & Overall sample $(\mathrm{n}=200)$ \\
\hline Mean age \pm SD & $44.79 \pm 14.56$ \\
\hline $\begin{array}{l}\text { Education (n; \%) } \\
\quad \text { Elementary school } \\
\text { Secondary school } \\
\quad \text { High school }\end{array}$ & $17(8.5 \%)$ \\
$\quad$ Master degree & $84(42.0 \%)$ \\
\hline Work status, n (\%) & $84(42.0 \%)$ \\
$\quad$ Student & $15(7.5 \%)$ \\
$\quad$ Worker & \\
$\quad$ Ron-worker & $1(1.0 \%)$ \\
\hline Mean height \pm SD & $90(45.0 \%)$ \\
\hline Mean weight \pm SD & $18(9.0 \%)$ \\
\hline Mean BMI \pm SD & $1.63 \pm 0.09$ \\
\hline
\end{tabular}

\section{Participants and Methods}

\section{Participants}

200 adult inpatients (162 women, 81\%) with severe obesity participated to this study. They were consecutively recruited at admission to the Division of Metabolic Diseases, Istituto Auxologico Italiano, Piancavallo (Verbania, Italy), where they were referred for a 3-week residential, multidisciplinary, integrated BRW program entailing energy-restricted diet, adapted physical activity as well as psychological and nutritional counseling [13]. Demographic and clinical characteristics of the sample are reported in table 1.

Inclusion criteria were age $\geq 26$ years and $\mathrm{BMI} \geq 35 \mathrm{~kg} / \mathrm{m}^{2}$ at the time of hospitalization, while exclusion criteria were the presence of major disorders of the central nervous system (e.g., epilepsy, dementia, or Parkinson's disease) and of any sensory, cognitive, or motor impairment that makes it difficult to fill in the study questionnaires.

The study was approved by the local Ethics Committee, and all participants provided written informed consent to participate.

\section{Procedure and Measures}

Immediately after admission to the Division of Metabolic Diseases, included inpatients were consecutively asked to fill in the following study questionnaires: PedsQL MFS for adults, FFS, and Center for Epidemiological Studies - Depression (CES-D). A random subset of 48 inpatients were asked to re-complete the PedsQL MFS after $72 \mathrm{~h}$ in order to test absolute and relative reliability.

\section{PedsQL MFS for Adults.}

The Italian version of the PedsQL MFS for adults was formally obtained from the MAPI Research Trust, and a user agreement was established with Dr. James W. Varni, licensor and author of the PedsQL tools. It was forward and backward translated by MAPI consultants in Italy and consists of 18 items that, according to the original conceptual model [14], compose 3 six-item scales: General Fatigue (e.g., 'I feel tired'; 'I feel too tired to do things that I like to do'), Sleep/Rest Fatigue (e.g., 'I feel tired when I wake up in the morning'; 'I rest a lot'), and Cognitive Fatigue (e.g., 'It is hard for me to keep my attention on things'; 'It is hard for me to remember what people tell me'). Responders are asked to rate how much of a problem each item has been during the past 1 month on a 5 -point Likert scale $(0=$ never, $1=$ almost never, $2=$ sometimes, $3=$ often, $4=$ almost always). According to the official scaling and scoring instructions, scale scores are computed by first reversing and linearly transforming the raw score of each item to a $0-100$ scale $(0=100,1=75,2=50,3=$ $25,4=0$ ), and then by averaging the transformed scores by the hypothesized dimensions. A total score is 
Manzoni et al.: Feasibility, Validity, and Reliability of the Italian Pediatric Quality of Life Inventory Multidimensional Fatigue Scale for Adults in Inpatients with Severe Obesity

Table 2. Items' descriptive statistics

\begin{tabular}{llllllll}
\hline & Mean & Median & SD & $\begin{array}{l}\text { Skewness } \\
\text { (standard error) }\end{array}$ & $\begin{array}{l}\text { Skewness } / \\
\text { standard error }\end{array}$ & $\begin{array}{l}\text { Kurtosis } \\
\text { (standard error) }\end{array}$ & $\begin{array}{l}\text { Kurtosis / } \\
\text { standard error }\end{array}$ \\
\hline Item 1 & 2.34 & 2 & 1.009 & $-0.208(0.172)$ & -1.209 & $-0.234(0.342)$ & -0.684 \\
Item 2 & 1.90 & 2 & 1.056 & $-0.005(0.172)$ & -0.029 & $-0.373(0.342)$ & -1.091 \\
Item 3 & 1.78 & 2 & 1.145 & $0.106(0.172)$ & 0.616 & $-0.757(0.342)$ & -2.213 \\
Item 4 & 1.42 & 1 & 1.118 & $0.368(0.172)$ & 2.140 & $-0.629(0.342)$ & -1.839 \\
Item 5 & 1.70 & 2 & 1.174 & $0.237(0.172)$ & 1.378 & $-0.849(0.342)$ & -2.482 \\
Item 6 & 1.71 & 2 & 1.159 & $0.017(0.172)$ & 0.099 & $-0.914(0.342)$ & -2.673 \\
Item 7 & 1.34 & 1 & 1.077 & $0.433(0.172)$ & 2.517 & $-0.529(0.342)$ & -1.547 \\
Item 8 & 1.75 & 2 & 1.344 & $0.177(0.172)$ & 1.029 & $-1.146(0.342)$ & -3.351 \\
Item 9 & 1.91 & 2 & 1.261 & $-0.057(0.172)$ & -0.331 & $-0.992(0.342)$ & -2.901 \\
Item 10 & 1.54 & 1 & 1.051 & $0.340(0.172)$ & 1.977 & $-0.425(0.342)$ & -1.243 \\
Item 11 & 1.11 & 1 & 1.049 & $0.765(0.172)$ & 4.448 & $-0.058(0.342)$ & -0.170 \\
Item 12 & 1.15 & 1 & 1.097 & $0.575(0.172)$ & 3.343 & $-0.636(0.342)$ & -1.860 \\
Item 13 & 1.49 & 2 & 1.056 & $0.233(0.172)$ & 1.355 & $-0.496(0.342)$ & -1.450 \\
Item 14 & 1.52 & 2 & 0.987 & $0.197(0.172)$ & 1.145 & $-0.379(0.342)$ & -1.108 \\
Item 15 & 1.25 & 1 & 0.990 & $0.433(0.172)$ & 2.517 & $-0.419(0.342)$ & -1.225 \\
Item 16 & 1.30 & 1 & 1.041 & $0.491(0.172)$ & 2.855 & $-0.283(0.342)$ & -0.827 \\
Item 17 & 1.36 & 1 & 1.046 & $0.391(0.172)$ & 2.273 & $-0.512(0.342)$ & -1.497 \\
Item 18 & 1.31 & 1 & 1.034 & $0.478(0.172)$ & 2.779 & $-0.263(0.342)$ & -0.769 \\
\hline
\end{tabular}

computed by averaging the responses to each item. However, with respect to the adult form, the original factorial structure (see model 1 in table 3 ) has not received any empirical support yet.

FFS

The Italian version of the FSS [11] was used in order to assess the convergent validity of the PedsQL MFS. It consists of 9 statements describing the negative effects of fatigue on motivation, exercise, physical functioning, the ability to carry out duties, work, family, or social life [15]. Responders are asked to rate each statement considering the previous week and using a Likert scale ranging from 1 (strong disagreement) to 7 (strong agreement). The total score is computed by averaging the raw scores of each item. The Italian version of the FSS was found to be psychometrically sound (valid, reliable, and responsive) in a recent study involving a sample of patients with obesity. In the present study, Cronbach's alpha resulted to be 0.94 (95\% CI 0.9-0.97), and the original, unidimensional, factorial structure was confirmed by the following fit values: RMESA $=0.079(90 \%$ CI 0.052-0.105), (RMSEA < 0.05) = 0.040; CFI = 0.966; $\mathrm{SRMR}=0.033)$.

CES-D

As for the FSS, the Italian version of CES-D [16] was used to evaluate the convergent validity of the PedsQL MFS. The CES-D consists of 20 items assessing affective, psychological, and somatic symptoms of depression [17]. Responders are asked to rate the frequency of the symptom described in each item on a Likert scale from 0 (rarely or none of the time) to 3 (most or all of the time). The CES-D has been shown to be a reliable measure for assessing the number, types, and duration of depressive symptoms across different racial, gender, and age categories, and it is characterized by good internal consistency (Cronbach's alpha coefficients ranging from 0.85 to 0.94 were reported in a recent review [18]). In the present study, Cronbach's alpha was 0.80 (95\% CI 0.75-0.86), and the original, unidimensional, factorial structure was confirmed by the following fit values: RMESA $=0.078$ (90\%CI 0.067-0.089), p $($ RMSEA $<0.05)<0.001$; CFI $=0.947$; WRMR $=1.027$.

\section{Statistical Analyses}

The factorial structure of PedsQL MFS was assessed by means of confirmatory factor analysis (CFA). In particular, four different models were fitted to the data and compared: i) the original three-factor model developed by Varni et al. [14]; ii) a modified three-factor model in which two items of the Sleep/Rest Fatigue scale (item 8 'It is hard for me to sleep through the night' and item 9 'I feel tired when I wake up in the 
Manzoni et al.: Feasibility, Validity, and Reliability of the Italian Pediatric Quality of Life Inventory Multidimensional Fatigue Scale for Adults in Inpatients with Severe Obesity

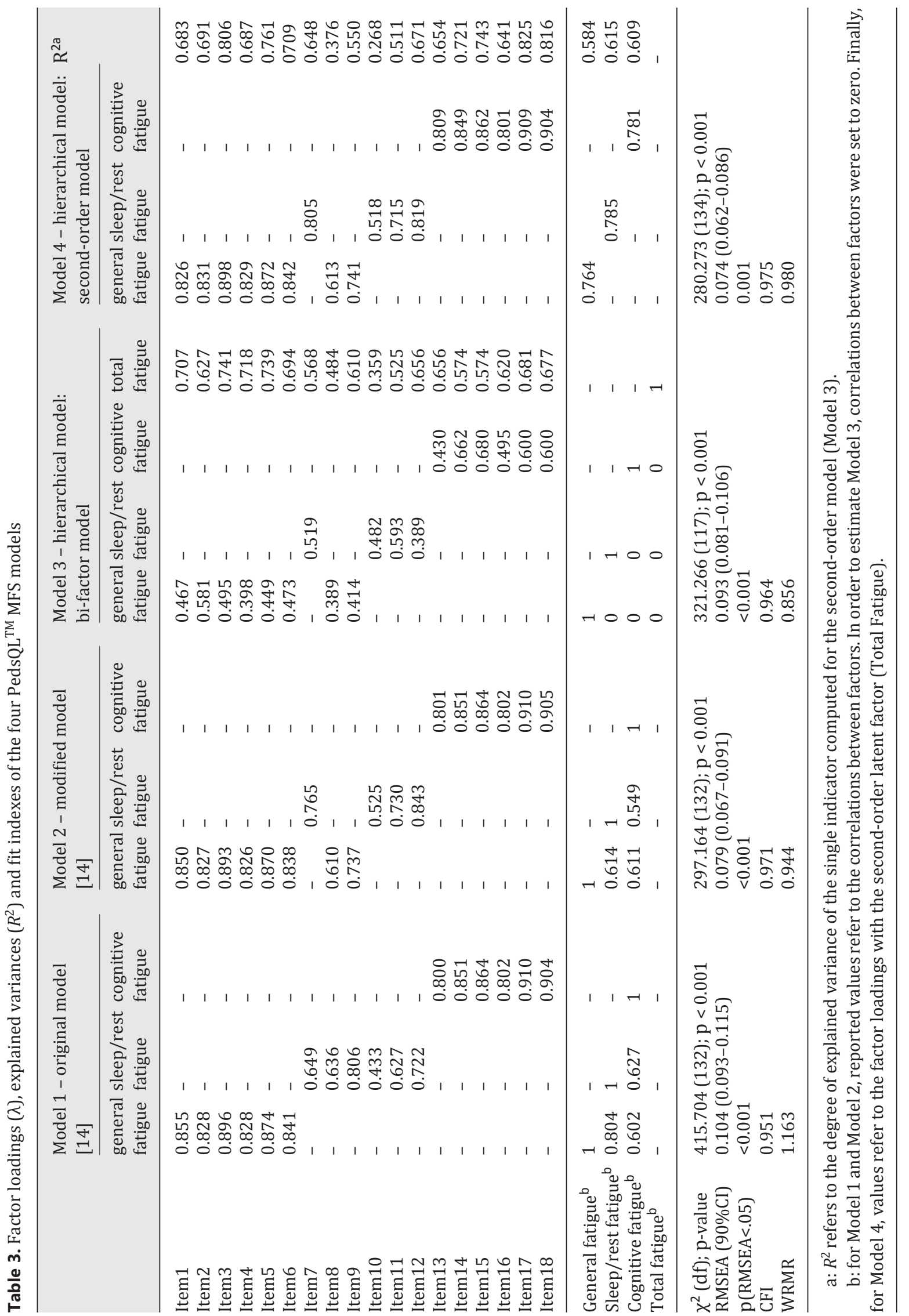


Manzoni et al.: Feasibility, Validity, and Reliability of the Italian Pediatric Quality of Life Inventory Multidimensional Fatigue Scale for Adults in Inpatients with Severe Obesity

morning') were allocated to load on the General Fatigue scale [14]; iii) a bi-factor model in which each item loads on a common first-order factor (Total Fatigue) and, simultaneously, on the respective first-order factors (General Fatigue, Sleep/Rest Fatigue or Cognitive Fatigue) as indicated by the best fitting model between 1 and 2; iv) a hierarchical model with a general second-order latent factor (Total Fatigue) over the three first-order factors (General Fatigue, Sleep/Rest Fatigue, or Cognitive Fatigue) as indicated by the best fitting model between 1 and 2. In this last model (Model 4), the correlations between the first-order factors are not freely estimated as in the first and second models but are accounted for by a second-order latent factor (Total Fatigue). This specification implies that the second-order factor is not defined by the items but by the three first-order factors (General Fatigue, Sleep/Rest Fatigue, and Cognitive Fatigue), which represent distinct latent sub-dimensions influenced by the broader construct of Total Fatigue [19, 20].

The second model was specified because Varni et al. [14], in a sample of university students and using Principal Component Analysis, found that item 8 and item 9 of the PedsQL MFS for young adults (18-25 years) did not load highest onto the pre-defined component (Sleep/Rest Fatigue) but onto the General Fatigue component. The 3rd and 4th models were instead specified and compared in order to assess the validity of the total score (Total Fatigue) and to define the most appropriate scoring procedure (Model 3 allows the computation of the total score by averaging all item scores, while in Model 4 the total score is computed by averaging the three sub-scales' scores).

The multivariate normality of data was assessed by means of the Mardia's skewness and kurtosis statistics, both of which resulted to be statistically significant. Univariate statistics are reported in table 2 and show that some items, in particular item 11 and item 12, had a skewed distribution, while some others, in particular item 8, were platykurtic. The mean- and variance-adjusted weighted least square (WLSMV) estimator with a polychoric correlation matrix computed on the raw scores was thus used. Model fit was assessed using the following indices: i) the root mean square error of approximation (RMSEA), with values between 0.05 and 0.08 suggesting a good fit of the model, and values below 0.05 indicating absolute fit; ii) the comparative fit index (CFI), with values greater than 0.95/0.96 indicating a good fit of the model; iii) the weighted root mean square residual (WRMR), with values of less than 1.0 suggesting a good fit; iv) the chi-square $\left(\chi^{2}\right)$ test, with $\mathrm{p}$ values greater than 0.05 test indicating the adequate fit of the model; and $v)$ the normed $\chi^{2}\left(\chi^{2} /\right.$ degrees of freedom), with values less than 2 indicating the adequacy of the model.

CFA was run using the R package 'Lavaan' (version 2.0-30).

Feasibility was assessed by computing the floor and ceiling effects, which were calculated on the raw scale scores as the percentage of patients reporting the lowest and the highest possible scores. The lowest and highest values were also calculated as the actual minimal and maximal scores plus or minus their corresponding minimal detectable change (MDC) (see 'Reproducibility and Internal Consistency' in 'Results' for the computation formulas). This procedure was applied for consideration of the measurement error [11].

Test-retest reliability was computed using the two-way mixed intra-class correlation coefficient $\left(\mathrm{ICC}_{\mathrm{co}}\right.$ nsistency), while agreement was determined by calculating the standard error of measurement (SEM $\mathrm{agreement})$ [21]. The minimal detectable change (MDC) was calculated as SEM $\times \sqrt{ } 2 \times 1.96$. Systematic bias was examined using the paired t-test. Internal consistency was calculated using the Cronbach's alpha coefficient.

The Pearson product-moment correlation coefficient was used to examine the hypothesized intercorrelations between the PedsQL MFS and the FSS, the CES-D or BMI. In particular, on the basis of the PedsQL MFS conceptual model it was expected that: i) the FSS would correlate significantly with all the PedsQL MFS scales but stronger with the scale reflecting the second-order factor, i.e. Total Fatigue; ii) BMI would correlate significantly with General Fatigue and Sleep/Rest Fatigue, but not with Cognitive Fatigue; and iii) CES-D would correlate significantly with all the PedsQL MFS scales. The strength of correlations was interpreted using the Cohen's benchmarks: <0.10 trivial; 0.10-0.30 small; 0.30-0.50 moderate; >0.50 large [24]. Moreover, the convergent validity of the PedsQL MFS was assessed by comparing patients after splitting them into two groups according to the CES-D clinical cut-off [16]. It was expected that patients reporting a raw score equal or higher than 24 and screening thus positive for a depressive disorder would have higher scores on all PedsQL MFS scales in comparison to patients with a CES-D score lower than 24. A t-test was used for this purpose. Finally, the convergent validity of the PedsQL MFS was also assessed by comparing patients with increasing amounts of comorbidities (4 levels: $0,1,2, \geq 3$ ). In particular, it was hypothesized that inpatients with more comorbidities would have also higher scores on the General Fatigue scale. A trend analysis (one-way ANOVA) was used for this purpose.

All analyses were run with the Statistical Package for the Social Sciences (SPSS) 19.0 for Windows (IBM, Armonk, , NY, USA) and the R software (release 3.1.2) using RStudio (version 0.99.441, RStudio Inc.). 
Table 4. Descriptive statistics of PedsQL ${ }^{\mathrm{TM}}$ MFS dimensions according to Model 4

\begin{tabular}{lcccllll}
\hline & Mean & Median & SD & $\begin{array}{l}\text { Skewness } \\
\text { (standard error) }\end{array}$ & $\begin{array}{l}\text { Skewness / } \\
\text { standard error }\end{array}$ & $\begin{array}{l}\text { Kurtosis } \\
\text { (standard error) }\end{array}$ & $\begin{array}{l}\text { Kurtosis / } \\
\text { standard error }\end{array}$ \\
\hline General fatigue & 54.72 & 54.69 & 22.872 & $0.026(0.172)$ & -0.149 & $-0.710(0.342)$ & -2.075 \\
Sleep/rest fatigue & 67.94 & 68.75 & 20.584 & $-0.527(0.172)$ & -3.064 & $-0.171(0.342)$ & -0.499 \\
Cognitive fatigue & 65.77 & 66.67 & 21.559 & $-0.310(0.172)$ & -1.803 & $-0.380(0.342)$ & -1.111 \\
Total fatigue & 62.81 & 63.71 & 17.772 & $-0.256(0.172)$ & -1.486 & $-0.093(0.342)$ & -0.273 \\
\hline
\end{tabular}

According to Varni and Limbers [14], item scores were linearly transformed on a scale ranging from 0 to 100.

\section{Results}

\section{Structural Validity}

Models 2 and 4 had the best fitting indices, and the likelihood ratio test on the difference between their respective chi-squares showed no significant difference between them (table 3). Model 1 showed the worst fit indices, followed by Model 3 whose RMSEA (0.093) and normed $\chi^{2}(2.75)$ were higher than the values indicating the adequacy of the model (table 3 ).

The PedsQL MFS scale scores (General Fatigue, Sleep/Rest Fatigue, and Cognitive Fatigue) that were used in the following analyses were thus computed by averaging the raw item scores according to Model 4. The total score was computed by averaging the three scale mean scores.

The factor loadings and fit indexes of all models are reported in table 3.

\section{Feasibility}

The lowest value (0) was reported by 3 patients on the General Fatigue scale, 9 on the Sleep/Rest Fatigue scale, 17 on the Cognitive Fatigue Scale, and 2 on the Total Fatigue Scale, while the highest value (4) was reported by only 1 patient on each of the three sub-scales. No participant scored highest on the Total Fatigue scale. Taking into account the MDC, the floor and ceiling effects were $16.5 \%$ and $6.5 \%$ on General Fatigue, $46.5 \%$ and $3.5 \%$ on Sleep/Rest Fatigue, $41.5 \%$ and $4.5 \%$ on Cognitive Fatigue, and $11.5 \%$ and $1 \%$ on Total Fatigue. All ceiling effects were thus lower than the $15 \%$ cut-off value considered acceptable [22, 23] but, with the exception of the Total Fatigue scale, all other floor effects were higher.

\section{Reproducibility and Internal Consistency}

Reliabilities as measured using the ICC were 0.89 (95\% CI 0.8-0.94) for General Fatigue, 0.73 (95\% CI 0.56-0.85) for Sleep/Rest Fatigue, 0.75 (95\% CI 0.59-0.86) for Cognitive Fatigue, and 0.85 (95\% CI 0.75-0.92) for Total Fatigue, while agreements as measured using the SEM were $0.30,0.41,0.38$ and 0.25 respectively. No significant systematic bias was found between test and re-test in any scale. The MDCs were 0.85 points for General Fatigue, 1.14 for Sleep/ Rest Fatigue, 1.06 for Cognitive Fatigue, and 0.7 for Total Fatigue. Cronbach's alphas were 0.91 (95\% CI 0.87-0.96), 0.77 (95\% CI 0.67-0.88) and 0.92 (95\% CI 0.86-0.97) for General Fatigue, Sleep/Rest Fatigue and Cognitive Fatigue respectively. The composite reliability index was computed for Total Fatigue and resulted to be 0.82 .

\section{Convergent Validity}

Significant but small positive correlations were found between age and General Fatigue (0.203), Cognitive Fatigue (0.148) or Total Fatigue (0.185), while significant and moderateto-large correlations were found between depression (CES-D) and all PedsQL MFS scales as 
Fig. 1. Convergent relationship between General Fatigue and the number of comorbidities.

Table 5. Pearson productmoment correlations between the PedsQL ${ }^{\mathrm{TM}}$ MFS scales and the convergent variables
Manzoni et al.: Feasibility, Validity, and Reliability of the Italian Pediatric Quality of Life Inventory Multidimensional Fatigue Scale for Adults in Inpatients with Severe Obesity

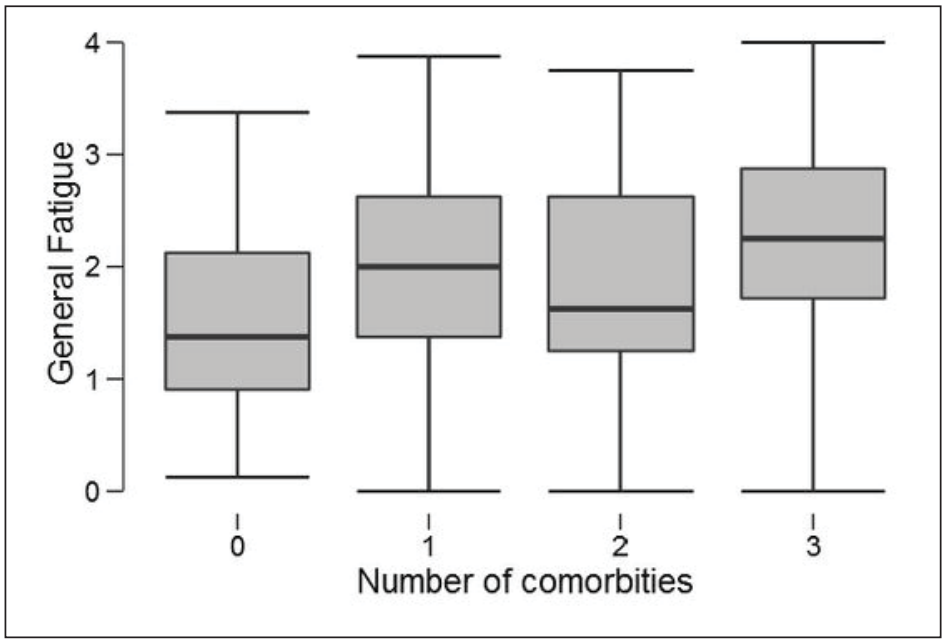

\begin{tabular}{lllll}
\hline & & CES-D & FFS & BMI \\
\hline 1 & Total fatigue & $0.758^{* * *}$ & $0.326^{* * *}$ & -0.032 \\
2 & General fatigue & $0.679^{* * *}$ & $0.396^{* * *}$ & 0.002 \\
3 & Sleep/rest fatigue & $0.405^{* * *}$ & $0.212^{* *}$ & 0.043 \\
4 & Cognitive fatigue & $0.767^{* * *}$ & $0.185^{* *}$ & -0.122 \\
\hline \multicolumn{3}{c}{$* * * \mathrm{p}<0.001 ;{ }^{* *} \mathrm{p}<0.010 ;{ }^{*} \mathrm{p}<0.050}$. \\
\hline
\end{tabular}

expected $(0.679,0.405,0.767$ and 0.758 for General Fatigue, Sleep/Rest Fatigue, Cognitive Fatigue and Total Fatigue respectively). Also the FSS was shown to correlate significantly with all PedsQL MFS scales (table 5) but, in partial contrast to our hypothesis, General Fatigue showed the highest correlation (0.423) instead of Total Fatigue (0.374). Unexpectedly, no significant correlations were found between the PedsQL MFS scales and BMI $(0.002,0.043$, -0.122 and -0.032 for General Fatigue, Sleep/Rest Fatigue, Cognitive Fatigue and Total Fatigue, respectively).

Patients screening positive for a depressive disorder on the CES-D $(n=91)$ had an higher mean score on all PedsQL MFS scales in comparison to patients screening negative $(n=109)$. In particular, the largest difference was found in the Cognitive Fatigue scale $(\mathrm{t}=-10.119, \mathrm{p}<$ $0.001 ; d=1.45)$, followed by Total Fatigue $(t=-9.263, p<0.001 ; d=1.32)$, General Fatigue $(\mathrm{t}=-8.489, \mathrm{p}<0.001 ; \mathrm{d}=1.21)$, and Sleep/Rest Fatigue $(\mathrm{t}=-3.736, \mathrm{p}<0.001 ; \mathrm{d}=0.52)$. Finally, significant linear and cubic trends were found between the number of comorbidities (4 levels: 0, 1, 2, $\geq 3$ ) and General Fatigue (fig. 1) as well as Total Fatigue (fig. 2).

\section{Transformation of Scores and Comparisons with Other Samples}

Scale scores were also computed according to the official scaling instructions, that is by reversing and linearly transforming the raw score of each item to a $0-100$ scale before averaging them by dimensions so that higher scores indicate better health-related quality of life (fewer symptoms of fatigue). Varni et al. [14] proposed this direct linear transformation 'for ease of interpretation'. As shown in table 4, both the means and the medians of all PedsQL MFStransformed scales are above the scale mid-point (50) but standard deviations are all around 20 , suggesting a large variance and limiting the interpretation of mean scores in absolute terms. 
Manzoni et al.: Feasibility, Validity, and Reliability of the Italian Pediatric Quality of Life Inventory Multidimensional Fatigue Scale for Adults in Inpatients with Severe Obesity

Fig. 2. Convergent relationship between Total Fatigue and the number of comorbidities.

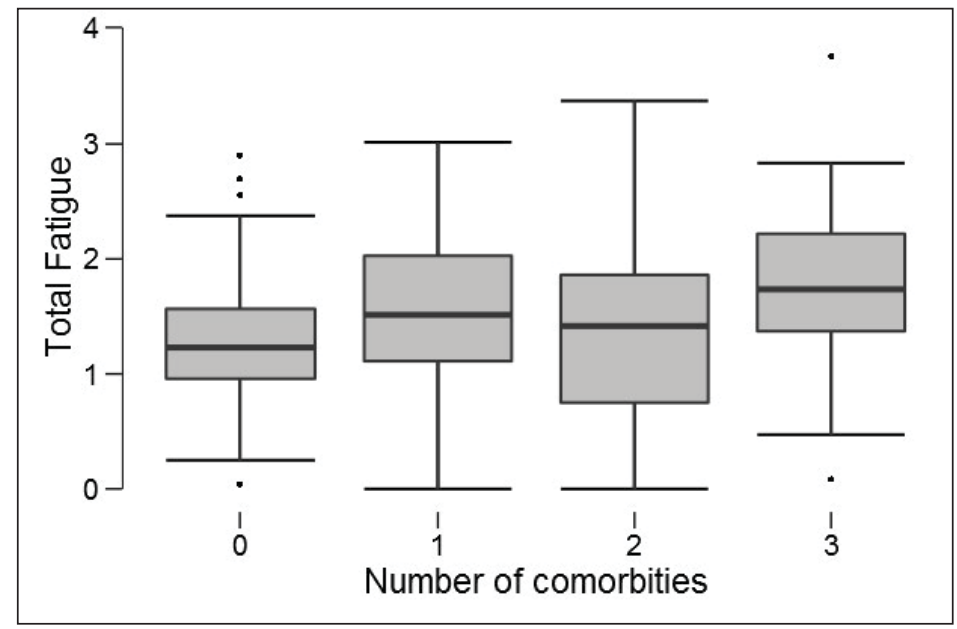

Furthermore, the distributions of both the Sleep/Rest Fatigue- and the General Fatigue-transformed scores significantly depart from normality: the former is negatively skewed and the latter is platykurtic. It is also important to consider that scale scores were calculated according to the modified model (table 3) and, with the exception of Cognitive Fatigue, the other scales' means could not be compared with the ones reported in previous studies using the PedsQL MFS with adults. However, there was no other way to examine the extent of fatigue (as measured with the PedsQL MFS) experienced by the inpatients with obesity included in the present study. The t-test was thus used to statistically assess differences in mean scores between the study sample and i) 202 healthy young adults aged 26-30 years [23], ii) 62 young adults aged 26-30 years with a chronic health condition [23], and iii) 64 long-term adult survivors of pediatric cancer with a mean age of $34.5 \pm 7.4$ years [24]. The Welch correction was used when comparing strongly unbalanced samples. Cognitive Fatigue resulted to be significantly lower in the first ( $p$ $=0.0001)$ and the third sample $(\mathrm{p}=0.0001)$, while Total Fatigue was found to be significantly lower in all three samples $(p=0.0001, p=0.0001$ and $p=0.0013$ for the first, the second and the third sample, respectively). An inverse result was found with respect to the General Fatigue scale because the respective mean score in the study sample was significantly higher (lesser fatigue) than in the sample of 62 young adults with a chronic health condition $(p=0.02)$. No statistically significant difference was found in the Sleep/Rest Fatigue scale.

\section{Discussion}

In the present study, the Italian version of the PedsQL MFS for adults (over 26 years old) was extensively examined in order to examine whether it is a valid, feasible, and reliable selfreport questionnaire for measuring the diverse domains of fatigue in inpatients with severe obesity.

First of all, the original factorial structure and the related scoring procedure were not supported in the present study. Instead, a modified hierarchical model with two items moved from the Sleep/Rest Fatigue domain ('It is hard for me to sleep through the night' and 'I feel tired when I wake up in the morning') to the General Fatigue scale and a second-order factor fitted the data well. Part of this result confirms what Varni et al. [14] found in a sample of university students by means of principal component analysis. In their study, the above mentioned items loaded highest onto the General Fatigue component, and they hypothesized that loss of sleep could be 'more related to feeling generally tired throughout the day and not 
Manzoni et al.: Feasibility, Validity, and Reliability of the Italian Pediatric Quality of Life Inventory Multidimensional Fatigue Scale for Adults in Inpatients with Severe Obesity

being able to finish tasks rather than taking naps or spending a lot of time in bed' (p. 112). This hypothesis was thus confirmed in this study and entails a change in the way the Sleep/ Rest Fatigue and the General Fatigue scores are computed: item 8 ('It is hard for me to sleep through the night') and item 9 ('I feel tired when I wake up in the morning'), originally developed to measure the Sleep/Rest Fatigue domain, should be definitively considered as part of the General Fatigue scale. A further modification of the original scoring procedure is determined by the new evidence of a second-order factor and also by the bad fit of the bi-factor model. The Total Fatigue score should not thus be computed by averaging the item scores but, as was done in this study, by averaging the mean scores of the three sub-scales.

With respect to feasibility, no missing response was detected, and ceiling effects were below the critical level (15\%) in all the PedsQL MFS scales. However, with the exception of the Total Fatigue scale, all other floor effects were higher than the percentage considered acceptable [22, 23], in particular for the Sleep/Rest Fatigue (46.5\%) and the Cognitive Fatigue $(41.5 \%)$ scales which, in inpatients with severe obesity, may have low sensitivities at low (or high if transformed) scores.

In order to examine the reproducibility of the PedsQL MFS scales, both reliability and agreement were assessed. All ICCs were close or higher than 0.8 , a value indicative of high between-subject discrimination in cross-sectional comparisons [21]. However, the 95\% CIs around the ICCs of the Sleep/Rest Fatigue and the Cognitive Fatigue scales have lower limits that are much below 0.8 , and this suggests that their reliabilities are significantly lower than the reliabilities of the General Fatigue and the Total Fatigue scales. Also agreements as measured using the SEM were quite different among the scales, with Sleep/Rest Fatigue showing the highest value and Total Fatigue the lowest one. In particular, on the basis of the raw scale (1-4), individual changes higher than 0.85 points on the General Fatigue scale, 1.14 points on the Sleep/Rest Fatigue, 1.06 points on the Cognitive Fatigue scale, and 0.7 points on the Total Fatigue scale can be interpreted as real and not due to measurement error with an acceptable probability level.

Overall, both the General Fatigue and the Total Fatigue scales demonstrated to be good in differentiating between inpatients with severe obesity and in detecting change over time in this population. The other two scales, i.e. Sleep/Rest Fatigue and Cognitive Fatigue, showed not to be as good as the previous ones, although their reproducibilities are acceptable anyway.

Internal consistency was excellent for both the General Fatigue and the Cognitive Fatigue scales, while the smallest but yet acceptable value was found for the Sleep/Rest Fatigue scale. This might depend on the lower number of items that, according to the best-fitting structural model (table 3), compose that scale (4 items). However, very similar Cronbach's alphas were found by Varni etal. [14] in a sample of University students using the original factorial structure ( 6 items for each scale). This means that moving two items of the Sleep/Rest Fatigue scale to the General Fatigue scale did not reduce the internal consistency of the former one and gives further support to the superior validity of the modified second-order model (table 3).

With the exception of the nonsignificant associations between all PedsQL MFS scales and BMI, all other hypothesized correlations were confirmed. The FSS correlated the most with General Fatigue and, to a bit lesser extent, with Total Fatigue. However, even if the correlations were close to the 0.50 cut-off, a value considered the minimum for showing adequate construct validity, a correlation of 0.50 means that only $25 \%$ of the variance is shared. Therefore, although the two questionnaires tap similar domains, they probably measured different aspects of fatigue in the present sample of inpatients with severe obesity. This is further supported by the significant but small correlations between the FSS and the other two PedsQL MFS scales, i.e. Cognitive Fatigue and Sleep/Rest Fatigue (table 5). Quite surprisingly, the highest correlations were found between General Fatigue, Cognitive Fatigue or Total Fatigue and depression (as measured with the CES-D). Indeed, fatigability, asthenia, and lack 
of energy are ancillary symptoms of several depressive disorders [24]. This result, together with the lack of correlations with BMI, might suggest that the PedsQL MFS General Fatigue, Total Fatigue and Cognitive Fatigue scales reflected a depressive condition rather than the physical impact of obesity. With respect to Cognitive Fatigue, this hypothesis is further supported by the nonsignificant association between the scale and the number of obesityrelated comorbidities which, otherwise, had a significant linear as well as cubic effect on General Fatigue and Total Fatigue. In fact, mean scores in both scales increase with the number of comorbidities, even if trends are flat between the category of patients with one comorbidity and the category of patients with two comorbidities (fig. 1, 2). This result showed that both scales, i.e. General Fatigue and Total Fatigue, did not measure mainly the symptoms of depression as the Cognitive Fatigue scale probably did, but reflected also the physical impact of the obesity-related comorbidities. The analysis of covariance (ANCOVA) showed indeed that trends were statistically significant, even after adjusting for depression (as measured with the CES-D). The nonsignificant correlations with BMI should thus not be interpreted as the evidence of a null effect of obesity on fatigue because, in the present sample of inpatients with severe obesity, the variance of BMI was small (6.05) and BMI is notoriously an imperfect measure of obesity which, however, exerted its effect on fatigue through its comorbidities.

Finally, the inpatients with severe obesity included in this study had an higher Total Fatigue in comparison with a sample of healthy young adults, a sample of young adults with a chronic health condition, and a sample of long-term adult survivors of pediatric cancer. Also Cognitive Fatigue was found to be higher in the inpatients with severe obesity than in healthy young adults and in long-term adult survivors of pediatric cancer. On the contrary, General Fatigue in obese inpatients was significantly lower than in a sample of young adults with a chronic health condition. However, these comparisons must be considered with caution due to the differences in the scoring of the PedsQL MFS Total Fatigue and General Fatigue scales and also due to the differences between the age of the inpatients with severe obesity included in this study and the age of the participants of the other studies'.

One important limitation of this study is indeed the lack of an age-matched healthy control group. This prevented the assessment of the known-group validity [14, 25] and limited also the appraisal of the fatigue extent experienced by the inpatients with severe obesity included in this study. Further limitations are the exclusively use of self-report measures to test convergent validity and the relatively small sample size of participants that re-completed the PedsQL $(\mathrm{n}=48)$.

\section{Conclusion}

This study supports a change in the scoring procedure of the PedsQL MFS for adults. Both the modified scales, i.e. General Fatigue, Sleep/Rest Fatigue and Total Fatigue, and the unchanged one, i.e. Cognitive Fatigue, resulted to be valid and reliable in a sample of inpatients with severe obesity, the only limitation being the low sensitivity of the Sleep/Rest Fatigue and the Cognitive Fatigue scales at low (or high if transformed) scores. Subsequent investigations should test the modified second-order model of the PedsQL MFS for adults in other samples and, with regard to patients with obesity, should assess its discriminant validity as well as its external responsiveness to weight reduction and to the improvement of both obesity-related comorbidities and depression.

\section{Ethical Approval}

All procedures performed in the study were in accordance with the ethical standards of the institutional research committee and with the 1964 Helsinki declaration and its later amendments. 
Manzoni et al.: Feasibility, Validity, and Reliability of the Italian Pediatric Quality of Life Inventory Multidimensional Fatigue Scale for Adults in Inpatients with Severe Obesity

\section{Funding}

The authors declare no financial or material support.

\section{Disclosure Statement}

The authors declare no conflict of interest.

\section{References}

1 Chaudhuri A,Behan PO: Fatigue in neurological disorders. Lancet 2004;363:978-88.

2 Valko PO, Bassetti CL, Bloch KE, Held U, Baumann CR: Validation of the fatigue severity scale in a Swiss cohort. Sleep 2008;31:1601-1607.

3 Chen MK: The epidemiology of self-perceived fatigue among adults. Prev Med 1986;15:74-81.

4 Varni JW, Limbers CA, Bryant WP, Wilson DP: The PedsQL multidimensional fatigue scale in pediatric obesity: feasibility, reliability and validity. Int J Pediatr Obes 2010;5:34-42.

5 Lim W, Hong S, Nelesen R, Dimsdale JE: The association of obesity, cytokine levels, and depressive symptoms with diverse measures of fatigue in healthy subjects. Arch Intern Med 2005;165:910-915.

6 Resnick HE, Carter EA, Aloia M, Phillips B: Cross-sectional relationship of reported fatigue to obesity, diet, and physical activity: results from the third national health and nutrition examination survey. J Clin Sleep Med 2006;2:163-169.

7 Lee YJ, Cho WJ, Kim JK, Lee DC: Effects of coenzyme Q10 on arterial stiffness, metabolic parameters, and fatigue in obese subjects: a double-blind randomized controlled study. J Med Food 2011;14:386-390.

8 Sartorio A, Fontana P, Trecate L, Lafortuna CL: Short-term changes of fatigability and muscle performance in severe obese patients after an integrated body mass reduction program. Diabetes Nutr Metab 2003;16:88-93.

9 Hjollund NH, AndersenJH, Bech P: Assessment of fatigue in chronic disease: a bibliographic study of fatigue measurement scales. Health Qual Life Outcomes 2007;5:12.

10 Elbers RG, Rietberg MB, van Wegen EE, Verhoef J, Kramer SF, Terwee CB, Kwakkel G: Self-report fatigue questionnaires in multiple sclerosis, Parkinson's disease and stroke: a systematic review of measurement properties. Qual Life Res 2012;21:925-944.

11 Impellizzeri FM, Agosti F, De Col A, Sartorio A: Psychometric properties of the Fatigue Severity Scale in obese patients. Health Qual Life Outcomes 2013;11:32.

12 Stein KD, Martin SC, Hann DM, Jacobsen PB: A multidimensional measure of fatigue for use with cancer patients. Cancer Pract 1998;6:143-152.

13 Sartorio A, Maffiuletti NA, Agosti F, Lafortuna CL: Gender-related changes in body composition, muscle strength and power output after a short-term multidisciplinary weight loss intervention in morbid obesity. J Endocrinol Invest 2005;28:494-501.

14 Varni JW, Limbers CA: The PedsQL Multidimensional Fatigue Scale in young adults: feasibility, reliability and validity in a University student population. Qual Life Res 2008;17:105-114.

15 Krupp LB, LaRocca NG, Muir-Nash J, Steinberg AD: The fatigue severity scale. Application to patients with multiple sclerosis and systemic lupus erythematosus. Arch Neurol 1989;46:1121-1123.

16 Fava GA: Assessing depressive symptoms across cultures: Italian validation of the CES-D self-rating scale. J Clin Psychol 1983;39:249-251.

17 Radloff LS: The CES-D scale: a self-report depression scale for research in the general population. Appl Psychol Meas 1977;1:385-401.

18 Carleton RN, Thibodeau MA, Teale MJ, Welch PG, Abrams MP, Robinson T, Asmundson GJ: The center for epidemiologic studies depression scale: a review with a theoretical and empirical examination of item content and factor structure. PLoS One 2013; 8:e58067.

19 Brown TA: Confirmatory Factor Analysis for Applied Research, 2nd ed. New York, Guilford Press, 2015.

20 Hoyle RH: Handbook of Structural Equation Modeling. New York, Guilford Press, 2012.

21 de Vet HC, Terwee CB, Knol DL, Bouter LM: When to use agreement versus reliability measures. J Clin Epidemiol 2006;59:1033-1039.

22 Mokkink LB, Terwee CB, Patrick DL, Alonso J, Stratford PW, Knol DL, Bouter LM, de Vet HC: The COSMIN study reached international consensus on taxonomy, terminology, and definitions of measurement properties for health-related patient-reported outcomes. J Clin Epidemiol 2010;63:737-745.

23 Terwee CB, Bot SD, de Boer MR, van der Windt DA, Knol DL, Dekker J, Bouter LM, de Vet HC: Quality criteria were proposed for measurement properties of health status questionnaires. J Clin Epidemiol 2007;60:34-42.

24 American Psychiatric Association (ed): Diagnostic and Statistical Manual of Mental Disorders, 5th ed. Washington, DC, American Psychiatric Association Publishing, 2013.

25 Haverman L, Limperg PF, van Oers HA, van Rossum MA, Maurice-Stam H, Grootenhuis MA: Psychometric properties and Dutch norm data of the PedsQL Multidimensional Fatigue Scale for Young Adults. Qual Life Res 2014;23:2841-2847. 